\title{
Carcinoma in situ de la mama: Controversias
}

\author{
Gildardo Gallego Noreña*
}

Recibido: Junio 8 I 2000

Revisado: Junio 29 I 2000

Aceptado: Agosto 11 I 2000

\section{RESUMEN}

Este artículo presenta los principales aspectos del cáncer in sito de la mama. Describe conceptos acerca de carcinoma lobular y ductal in sito de la mama. El cáncer lobulillar in situ es solo un marcador de un futuro cáncer. En cambio el carcinoma ductal in situ es un verdadero predictor de un cáncer invasivo en el futuro.

La participación de la paciente para tomar decisiones acerca de su tratamiento es muy importante. Cada caso debe mirarse en forma independiente y hoy existe una tendencia a realizar tratamientos conservadores y no cirugías radicales. Existen razones precisas para realizar mastectomías, cuadrantectomías o resecciones locales.

Se enfatiza la importancia de las márgenes o bordes libres cuando se efectúa tratamiento conservador de esta manera se garantiza el éxito del tratamiento. El porcentaje de recurrencia es el mismo cuando se usa tratamiento conservador o radical.

PALABRAS CLAVES: Cáncer lobulillar y ductal in sito. Tratamiento conservador de cáncer de mama.

\section{SUMMARY}

This article presents the principal aspects of in sito breast cancer, its describes concepts about lobulillar and ductal in sito breast cancer.

The lobuliliar cancer is only a marker of roture breast cancer. The ductaI cancer in sito is a predictor of invasive cancer in the roture. The participation of a patient to take decisions about their treatment is very important. Each case would be saw in a independent way and today there are tendency to conservative treatment and no radical surgeries. There are precise indications to perform mastectomy or conservative treatments as cuadrantectomy or local resections.

It emphasizes the importance of free margin when we perform conservative manner, thus guarantee the success of treatment. The recurrence percentage as the same when we use conservative or radical treatment.

KEY WORDS: LobulilIar and ductal cancer in sito, conservative breast cancer.

\section{Historia}

El carcinoma in situ de la mama ha sufrido cambios substanciales a medida que se conoce más su evolución y su comportamiento biológico. El tratamiento radical también se ha modificado con el paso de los años, se ha sustituido por terapias menos agresivas y más conservadoras. La conceptualización y definición de puntos claves en el entendimiento del cáncer mamario han consolidado su manejo. Antes de la época de 1985 toda paciente con cáncer in situ era sinónimo de mastectomía radical, hoy con la modalidad de tratamientos conservadores para carcinomas invasivos, parecería ilógico no ofrecer esta alternativa para el cáncer in situ.

\section{Variedades}

El cáncer in situ de la mama comprende: carcinoma lobulillar o lobular in situ, carcinoma ductal in situ y la enfermedad de Paget del pezón.

\section{Carcinoma lobular o lobulillar in situ (CLIS)}

Algunos lo consideran como una lesión precancerosa, lo han denominado neoplasia lobulillar o lobular. Fue descrito por primera vez por Foote y Stewart en 1941, constituye entre el 3 a15\% de los carcinomas de la mama. Su concepción ha cambiado de acuerdo con el entendimiento de la biología molecular y mejor observación de la entidad. En el momento actual se considera como un simple marcador de riesgo de padecer un carcinoma invasor con el paso de los años (1).

Se diagnostica el carcinoma lobulillar in situ de 5 a 15 años antes que se desarrolle un carcinoma invasor. Un $25 \%$ de los lobulillares in situ, evolucionarán a un carcinoma invasor. La observación más curiosa en cuanto al CLIS es que la lesión invasora predominante que aparece más tarde no es un lobulillar invasor sino un ductal invasor.

El carcinoma lobulillar in situ tiene una multicentricidad de 90 por ciento y un 70 por ciento de bilateralidad, por 10 tanto una resección local amplia no solucionaría lo

* $\quad$ Profesor Emérito. Universidad de Antioquia. 
primero y una mastectomía tampoco solucionaría lo segundo. El riesgo de carcinoma lobular invasor, después de una biopsia, es el mismo en la mama ipsilateral que en la contralateral. No se encuentran metástasis a no ser que se vincule con tumor invasor concomitante.

Se piensa que la incidencia es alta con relación a otros carcinomas porque todos los acinos están sometidos a procesos y estímulos similares.

Teniendo en cuenta los tipos histológicos su frecuencia está entre 2 y 5 por ciento, presentándose entre los 40 y 46 años, es decir tres cuartas partes de las mujeres son menopáusicas (2-3). Desde el punto de vista macroscópico no ofrece manifestación alguna, se asocia frecuentemente con mastopatía fibroquística.

Si se considerara como una verdadera enfermedad no tiene manifestaciones clínicas ni mamográficas, permanece silencioso y solo 25 por ciento evolucionan a un carcinoma invasor. La incidencia aumentó por mejoras en la mamografía, por la prescripción generalizada de ésta, y por mayor conciencia para biopsiar lesiones sospechosas demostradas por la misma mamografía, en ocasiones el único hallazgo es la presencia de microcalcificaciones.

En resumen la entidad es un marcador del desarrollo de un futuro cáncer invasor, un 25 a un 30 por ciento de las pacientes desarrollarán carcinoma- invasor en el curso de unos 20 años posterior. El carcinoma invasor subsecuente puede ser un carcinoma lobulillar o un ductal. Varios investigadores han seguido esta entidad por años y han demostrado la ocurrencia de carcinoma invasor en porcentajes que van desde el7 hasta el 30 por ciento. Con el fin de evitar esta ocurrencia se recomienda una vigilancia cuidadosa estricta, así el riesgo de morir por esta causa es mínimo. La conducta de observación se recomienda cuando la lesión mamo gráfica no es muy extensa y no halla otras lesiones, además la paciente debe ser de fácil seguimiento. La vigilancia debe ser con autoexamen mensual, examen clínico cada seis meses y mamografía semestral por los dos primeros años, además, quimio- prevención con antiestrógenos, (4-5) concepto que no es compartido por muchos investigadores.

\section{Carcinoma docta) in sito (CADIS)}

Broders en 1932 lo define como la transformación maligna de las células del epitelio ductal y que permanece confinado al mismo sin presentar ruptura de la membrana basal. Por definición no puede entrar o penetrar a los canales linfáticos o vasculares y por 10 tanto no da metástasis.

En la evolución del carcinoma mamario el ductal in situ es un fenómeno local y no una enfermedad sistémica. Se sugiere que el CADIS es un paso en la progresión del tejido normal al cáncer invasor y se considera un verdadero precursor anatómico del carcinoma in vasor de la mama.

Diagnóstico: se diagnostica casi generalmente en forma accidental por hallazgo histopatológico de una biopsia de mama realizada por lesión nodular palpable, o se diagnostica por descarga sanguinolenta por el pezón o por el hallazgo mamo gráfico de microcalcificaciones, o por alteraciones del pezón que hacen sospechar enfermedad de Paget (6). Más del 70 por ciento de los carcinomas in situ se acompañan de microcalcificaciones (6). Las microcalcificaciones se deben a las precipitaciones de calcio en los conductos mamarios, además se asocian con detritus celulares y necrosis tisular, la cual es más frecuente en el tipo comedocarcinoma (que tiene necrosis central). Las microcalcificaciones están presentes además del comedo en el carcinoma cribiforme y menos común en el micropapilar (3). Cualquier grupo que contenga más de cinco microcalcificaciones en cúmulo es indicativo de biopsia. La punción con aguja fina (BACAF), no debe considerarse una prueba confiable para diagnosticar carcinoma in situ. También puede diagnosticarse el CADIS en nódulos bien circunscritos no palpables.

Antes de la mamografía se diagnosticaba el carcinoma ductal in situ (CADIS) en 1 ó 2 por ciento, y ahora con el uso de la mamografía sistemática, se diagnostica o sospecha entre el 20 y 40 por ciento, hasta el punto de que en 1997 en EEUU se habían diagnosticado 3.000 casos nuevos de CADIS (4). Los programas de tamizaje bien llevados demostrarán en las mamografías con mayor frecuencia nódulos indeterminados, microcalcificaciones en cúmulos con patrón ductal, distorsi6n, etc., lesiones que no son palpables y son sugestivas de carcinoma in situ. Es necesario considerar que la mamografía en la fase preclínica del carcinoma es poco específica, pues tan solo el 30 por ciento de las lesiones sospechosas no palpables son verdaderos carcinomas. En muchas ocasiones la imagen mamográfica no guarda correlación con el diagnóstico histológico final.

Una vez que se considera sospechosa una mamografía, se realiza biopsia de las lesiones no palpables mediante. localizaci6n con aguja o con arp6n. El fragmento extirpado marcado con aguja se somete a control radiológico con el fin de determinar la zona en la cual el mayor número de calcificaciones y de ahí realizar el mayor número de secciones histológicas.

Existen algunas opiniones que pueden ser contradictorias sobre el carcinoma in situ que han sido bien documentadas, así: en el $50 \%$ de los casos con biopsias positivas de carcinoma in situ no se encontró lesión residual en las mastectomías. El carcinoma in situ es bilateral en ellO al $20 \%$ de los casos y es multicéntrico en el 60\%. En el sitio de biopsia de carcinoma in situ al cabo de algunos años se desarrolla carcinoma invasor en un 25 por ciento de los casos.

Anatomía Patol6gica: se cree que el carcinoma ductal in situ es un grupo de enfermedades heterogéneas en el cual existen varios tipos arquitectónicos como el s6lido, cribiforme, comedocarcinoma, papilar, micropapilar, de células claras y el clinging. Recientemente se las agrupa en lesiones tipo comedo y no comedocarcinoma (6-7). La clasificaci6n del carcinoma ductal in situ por subtipos es importante porque en conjunto con otros factores pronósticos puede ayudar a tomar decisiones terapéuticas. Algunos de los factores pronósticos son muy conocidos como: tamaño de la lesi6n, subclasificaci6n histopatol6gica, grada de diferenciaci6n celular, reacci6n fibrosa perilesional, grado de angiogénesis, etc.

Las lesiones tipo comedocarcinoma son pleomórficas, de alto grado nuclear, con numerosas mitosis, necrosis central y parecen histológicamente más malignas. En los focos de necrosis hay calcificaciones que se observan por 
radiología. La visión macroscópica demuestra engrosamiento tisular que a la expresión puede secretar material espeso y blanco amarillento como el que se observa en los comedones, su aspecto es similar a la comedomastitis. Cuando se identifica esta lesión es necesario incluir numerosos cortes con el fin de descartar invasión. También las lesiones de tipo comedocarcinoma expresan mayormente HER2/NEU, tienen alta rata proliferativa medida por timidina marcada y tendencia a microinfiltración. (6- 7, 11, 13). El alto grado nucleares la clave histopatológica para identificar el comportamiento agresivo: las lesiones de tipo no comedocarcinoma tienden a ser lo opuesto. El problema de clasificarlas como comed o y no comedocarcinoma es que los patólogos no tienen un acuerdo sobre terminología actual. Se piensa que el grado nuclear es de gran importancia, es mejor marcador biológico que la misma arquitectura y es la llave histológica para expresar agresividad.

Clasificación según el grado nuclear: el grado nuclear según varios autores da la clave para clasificar el CADIS en lesiones de bajo grado y alto grado; las lesiones de bajo grado se

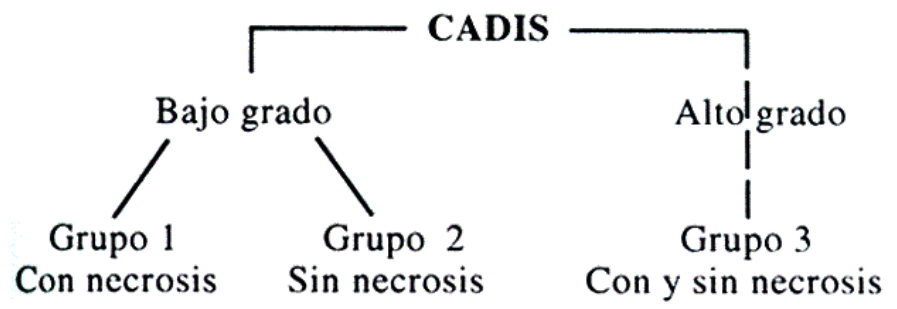

las que presentan necrosis. Las lesiones de alto grado nuclear constituyen el grupo 3 y pueden tener o no necrosis (8). La representac1ón esquemática sería:

Para tomar la decisión lo más correctamente posible en cuanto a tratamiento conservador, se prefiere en la actualidad aplicar el Indice Pronóstico de Van Nuys (VPNI).

Indice Pronóstico de Van Nuys (VPNI): el centro de patología mamaria en la ciudad de Van Nuys en California liderado por Douglas Marchant,(8) idean un índice pronóstico en el cual analizan una cantidad de factores con el fin de servir de guía para tomar una decisión terapéutica. Se sugiere que el patólogo debe estar presente en la sala de cirugía para recibir y orientar la muestra, precisar la extensión de la lesión y caracterizar márgenes. Los factores clínicos, patológicos y de laboratorio, pueden usarse como ayuda al clínico y a las propias pacientes con el fin de tomar una decisión en cuanto a su tratamiento.

Varios investigadores muestran que el grado nuclear, el tamaño del tumor, la presencia de comedonecrosis, el estado de las márgenes del tumor, son factores importantes en predecir la recurrencia local en pacientes con CADIS, quienes eligen conservar la mama $(7,9)$. Usando la combinación de estos factores es posible seleccionar grupos de pacientes que no requieren irradiación, y otro grupo de pacientes que presentan rata elevada de recurrencia, aún con irradiación, y en quienes se les aconseja la mastectomía.
El VNPI sale de la combinación de tres predictores estadísticamente significantes (por análisis multivariado) de recurrencia local en pacientes con carcinoma ductal in situ. Estos tres factores son: tamaño del tumor, márgenes (bordes) del tumor y clasificación patológica. El score 1 es buen pronóstico y el 3 peor pronóstico. Cada factor tiene gradación de 1 a 3 . Con los tres predictores se agrupó las pacientes en tres grupos y se usó la recurrencia local como un marcador de falla del tratamiento (8).

Índice de Van Nuys:

Score 1 2 3

$\begin{array}{llcc}\text { Tamaño Tumor mm } & <15 & 16-40 & >41 \\ \text { Márgenes mm } & >10 & 1-9 & <1 \\ \text { Clasificación } & \begin{array}{l}\text { Bajo } \\ \text { grado }\end{array} & \begin{array}{c}\text { Bajo } \\ \text { grado }\end{array} & \begin{array}{r}\text { Alto } \\ \text { grado }\end{array} \\ \text { Patológica } & \begin{array}{l}\text { sin } \\ \text { Necrosis }\end{array} & \begin{array}{c}\text { con } \\ \text { necrosis }\end{array} & \begin{array}{r}\text { con-sin } \\ \text { necrosis }\end{array}\end{array}$

El mejor VNPI es de 3 con scores de 1 para cada predictor y el peor VNPI fue de 9 con scores de 3 para cada predictor. Se incluirá en este último lesiones mayores de $40 \mathrm{~mm}$, con lesiones positivas y lesiones histológicamente de alto grado.

Pacientes con carcinoma ductal in situ y VNPI de 3 son pacientes que tienen poca o nula probabilidad de recurrencia local y por lo tanto no requieren irradiación. Pacientes con Carcinoma ductal in situ y VNPI de 1, 6, 7, pueden tener rata intermedia de recurrencia local, se pueden beneficiar de reexcisión, y si el score permanece en este rango, se recomienda la irradiación. En cambio pacientes con score de 8, 9 tienen una recurrencia extremadamente elevada, y se recomienda. por lo tanto. una mastectomía con reconstrucción inmediata (8).

\section{Tratamiento}

En este momento que se proclama el tratamiento conservador para lesiones pequeñas de cáncer invasor es ilógico insistir en mastectomías para lesiones de carcinomas ductales in situ. Hasta el año de 1980 el tratamiento ideal para el CADIS era la mastectomía, (6) ahora con mejor conocimiento del comportamiento biológico, y las mejoras técnicas de la mamografía, además participación de la paciente en la toma de decisiones, surgen modalidades terapéuticas conservadoras con perspectivas más promisorias en el enfoque dinámico de esta entidad (2-3, 10-12).

Los carcinomas ductafes in situ tipo no comedocarcinoma se pueden tratar con resección local amplia, siempre y cuando esta resección tenga márgenes libres. Algunos centros acostumbran realizar biopsia por congelación de los bordes de la lesión extirpada, cuando estos están comprometidos se puede realizar una reexcisión. Hasta donde sea posible se aplicará el índice de VNPI, de 
acuerdo con este índice se adiciona radioterapia complementaria. La radioterapia se aplica a toda la mama y además se da refuerzo al lecho tumoral.

Indicaciones de mastectomía: pacientes que presentan CADIS y no aceptan tratamiento conservador o tienen algún factor de riesgo para recurrencia local $(3,9,15)$.

Algunas características están ya muy definidas para que se tome la decisión de mastectomía en pacientes con CADIS.

Se recomienda mastectomía en casos de CADIS cuando: 1. Existen microcalcificaciones difusas en la mamografía o la lesión es multicéntrica, 2. Cuando existe una área nodular mayor de 4-5 centímetros de diámetro, 3. Cuando hay dificultad para conseguir márgenes libres, 4. En los casos de comedocarcinoma, 5. En pacientes de difícil seguimiento y 6 . Cuando la paciente expresamente pida la mastectomía.

Las pacientes sometidas a mastectomía para carcinoma in situ se recomienda en la actualidad reconstrucción mamaria inmediata, también denominada terapia de reemplazo glandular (14). Como el carcinoma in situ no invade la piel no es necesario realizar extirpación amplia de piel, así como tampoco es necesario realizar vaciamiento axilar puesto que no hay metástasis, pero algunos investigadores recomiendan el vaciamiento axllar o un muestreo ganglionar por si existiese la posibilidad de microinvasión.

\section{Conclusiones}

Es necesario tener claro el conocimiento de lo que es el carcinoma in situ de la mama, saber su comportamiento con el fin de definir la terapia a seguir.

El carcinoma lobular in situ no es un verdadero carcinoma como tal sino un simple marcador, predice el posible riesgo de aparición de un carcinoma lobular invasor. Por lo tanto la terapia consiste únicamente en observación clínica y mamográfica.

El porcentaje creciente de tumores detectados por mamografía, el menor tamaño de las lesiones invasoras, el desarrollo de indicadores pronósticos mas precisos como la determinación de la citometría de flujo, estudios de fase $\mathrm{S}$, análisis de oncogenes indican que procedimientos conservadores se implementen con mayor seguridad. Es así como poco a poco se trata de aplicar índices pronósticos como el de Van Nuys con criterios muy definidos para clasificar las pacientes en terapias conservadoras, complemento con radioterapia o mastectomías si es del caso.

El médico debe explicar y destinar parte de su tiempo para presentar con claridad las posibles alternativas terapéuticas a la paciente, debe permitir que ella participe en las decisiones para evitar reproches ulteriores al cirujano en caso de que se desarrollen recidivas. La cirugía radical (mastectomías) tiene sus indicaciones muy precisas, lo mismo que la cirugía conservadora.

\section{BIBLIOGRAFIA}

1. Fisher Edwin. Pathobiological considerations relating to the treatment of intraductal carcinoma (ductal carcinoma in situ) of the breast, Ca cancer J Clin 1996; 47: 52-64.

2. McNelis John, MD, Wise L. Carcinoma in situ, Clin Obst y Gin

3. de Norte América 1994; 2: 917-920.

4. Guías de manejo de tumores marnarios. Revista Colombiana de Obstetricia y Ginecología 1998; 48(4).

5. González J Merlo y cols.: Oncología Ginecológica, Salvat Editores, Mallorca, Barcelona, 1991; 379-542.

6. Alvarez GE, Tejerina Gómez A. Mastología Dinámica, Díaz de Santos S.A. Madrid, 1995.

7. Hetelekidis S, Schnitt S. Morrow M, Harris J. Managements of ductai carcinoma in situ Ca Cancer J Clin 1995; 45: 244-253.

8. Hughes KS, Lee AK, Rolfs A. Controrversies in the treatment of Ductal Carcinoma in Sitl: Surgicai Clinics of North America. 1996; 76(2): 243265.
9. Marchant DJ. Breast Disease. W.B. Saunders Company, Philadelphia Pennsylyania: 1997; 163-195-213.

10. López MI. Conceptos actuales en el manejo del cáncer mamario, Trib Med 1992; 85: 117-133.

11. Petrek I. Cáncer mamario, Clin Obst y Ginec 1994; (4): 885-896. 11. Hortobagyi GN, Buzdar AV. Current status of adjuvant systemic therapy for primary breast cancer: Progress and controyersy. CA Cancer I Clin 1995; 45: 199-226.

12. Gutiérrez R. Cáncer del seno: su tratamiento quirúrgico primario, Rey Col de Cirugía 1993; 8(1): 37-44.

13. Torres Trujil10 R. Tumores de Mama. primera edición, México, Interamericana, Mcgraw Hill, 1994.

14. Maldonado H. Tratamiento conservador del cáncer mamario. Rey Col de Cirugía. 1993; 8(1): 30-36.

15. Perry F. Rey Col de Obst y Gin 1995; 46(1): 11-15. 